FERMILAB-TM-2750-AD

Fermilab IOTA/FAST Run 2 Experimental Report Beams-doc-8837

\title{
Report on Single and Multiple Intrabeam Scattering Measurements in IOTA Ring in Fermilab*
}

\author{
V.A. Lebedev ${ }^{\dagger}$ I. Lobach, A. Romanov, A. Valishev \\ Fermi National Accelerator Laboratory, PO Box 500, Batavia, Illinois 60510, USA
}

\section{Abstract}

The study of single and multiple intrabeam scattering was carried out in the IOTA ring at Fermilab. The report discusses the results of the measurements and outlines still unresolved problems

Keywords: Intrabeam scattering, residual gas scattering, Touschek scattering

- This manuscript has been authored by Fermi Research Alliance, LLC under Contract No. DE-AC02-07CH11359 with the U.S. Department of Energy, Office of Science, Office of High Energy Physics.

†val@,fnal.gov 


\section{Contents}

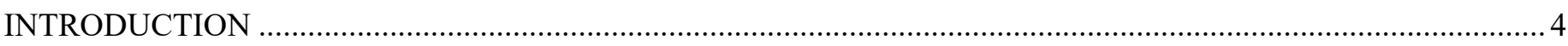

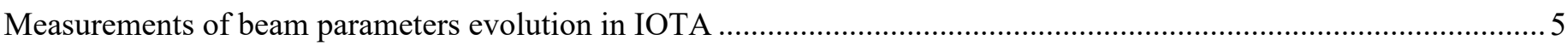

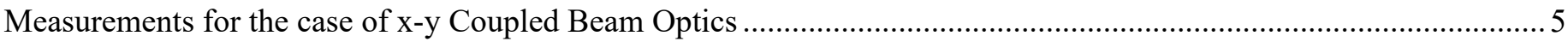

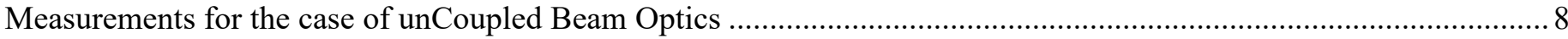

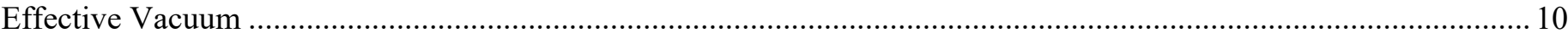

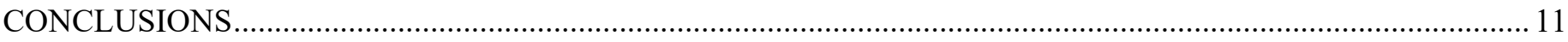

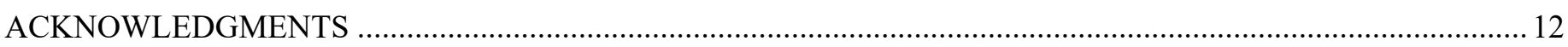

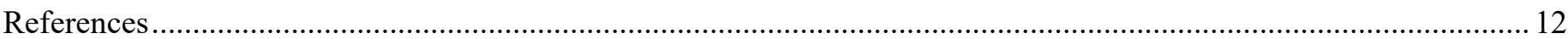




\section{INTRODUCTION}

The study of single and multiple intrabeam scattering (IBS) was carried out in the IOTA ring at Fermilab. The goals and objective of this study were:

(1) Verify accuracy of IOTA instrumentation; in particular: measurements of beam emittances, bunch length, momentum spread, bunch and beam currents

(2) Optics verification as a byproduct of beam size measurements

(3) Measurements and calibration of RF voltage which also include the measurements of beam deceleration due to synchrotron radiation, interaction with vacuum chamber and RF cavities

(4) Characterization of vacuum which is very important for the coming experiments in Optical stochastic cooling (OSC)

The above measurements and parameters are related through the theories of multiple IBS, Touschek scattering, and scattering at the residual gas. At large beam currents the measurements were affected by storage of residual gas ions in the electron beam.

Thus, these experiments helped us to verify and refine the theoretical models used in calculations of IBS, and elastic and inelastic gas scattering. 


\section{MEASUREMENTS OF BEAM PARAMETERS EVOLUTION IN IOTA}

The measurements were acquired at two shifts. The first one was done at March 6, 2020. It included the measurements of beam parameters evolution in the uncoupled and fully $(100 \%)$ coupled lattices. The second set of measurements was acquired at March 17, 2020. It includes the measurements in the uncoupled lattice only. Note that two data sets for uncoupled measurements still have somewhat different coupling because coupling in the second measurements (March 17) was not suppressed as good as for the first measurement.

All data were archived in the IOTA archivers and are available for additional analysis if required.

To accelerate the measurements the beam was scraped time-to-time because the beam parameters evolution was greatly slowed down with beam current reduction.

The data analysis pointed out that the model of multiple scattering at the residual gas requires significant improvements. This work has been recently carried out and will be reported and published in the coming months.

The study results are documented in the presentation at the IOTA collaboration meeting [1]. Figure 1 presents the beam current measurements for the March 6 data.

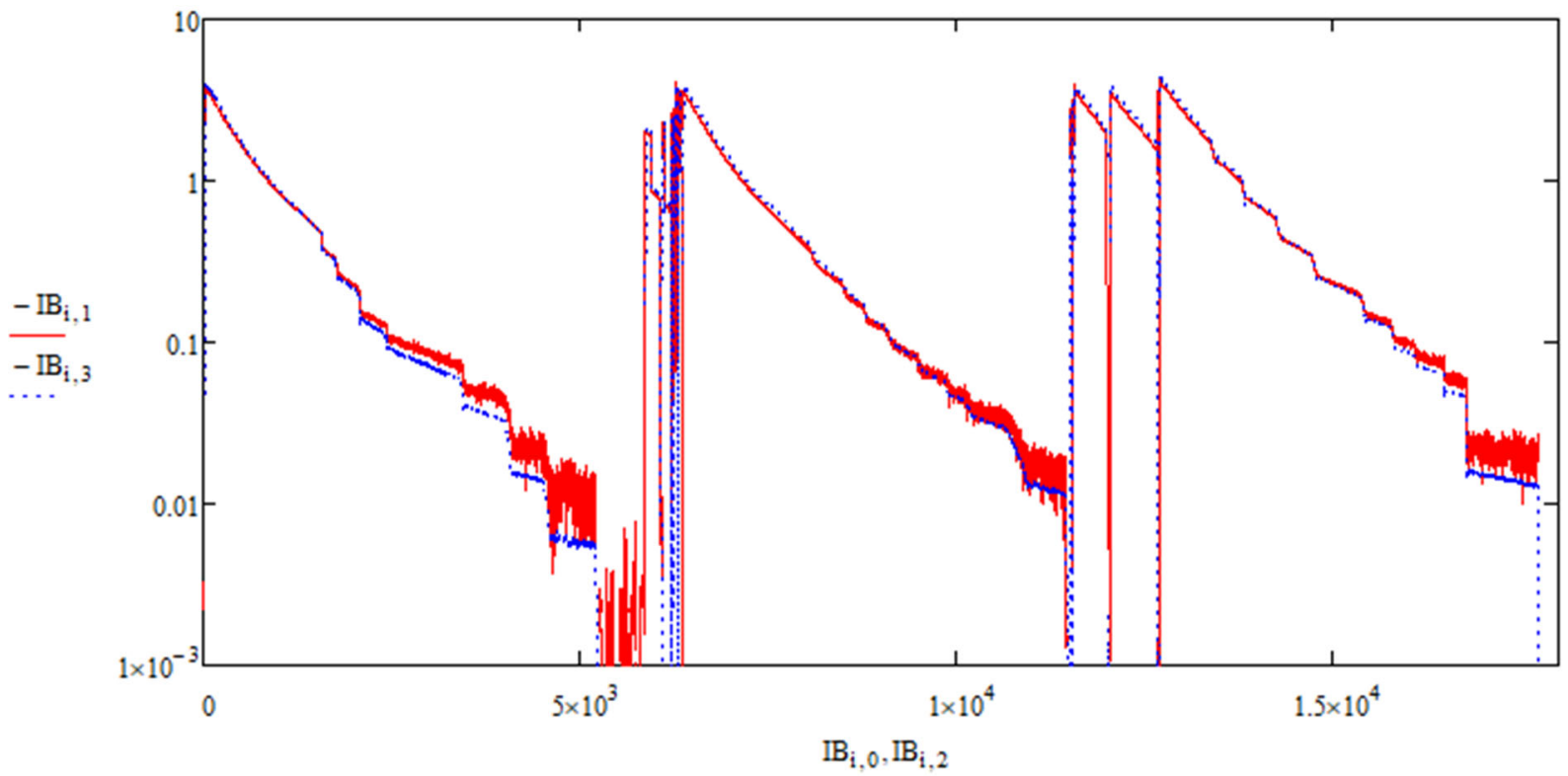

Figure 1: Beam current from DCCT (red) and WCM (blue) for 3 measurement of March 6: first two-uncoupled beam, the last one-measurements in the $x-y$ coupled optics.

\section{MEASUREMENTS FOR THE CASE OF X-Y COUPLED BEAM OPTICS}

Figure 2 presents coupled beta-functions for the beam optics used in the measurements. After careful decoupling the coupling was introduced by changing current in SQA1L skew-quad by GdL $=200 \mathrm{G}$. That corresponded the tune split of 0.015. The major ring parameters are:

- Damping parameters: $\mathrm{g}_{1}=0.685, \mathrm{~g}_{2}=.678, \mathrm{~g}_{\mathrm{s}}=2.636$

- Equilibrium rms emittances set by synchrotron radiation (SR): $\varepsilon_{1}=12.1 \mathrm{~nm}, \varepsilon_{2}=13.0 \mathrm{~nm}$.

- Equilibrium relative rms momentum spread set by SR: $\sigma_{\mathrm{p}}=8.75 \cdot 10^{-5}$

- Emittance damping time: $\tau_{1}=3.51 \mathrm{~s}, \tau_{2}=3.54 \mathrm{~s}, \tau_{\mathrm{s}}=0.91 \mathrm{~s}$

The theoretical analysis of the beam parameters evolution was based on the equations for fully coupled optics [2]. The 4D-Twiss parameters used in computation of single and multiple IBS [3]. The analysis assumed non-relativistic beam in the beam frame. Although the electron velosities in the beam frame grow with the beam current they still stay non-relaticistic even at highest beam current of $4 \mathrm{~mA}$ used in the measurements. The standard approximation for non-relativistic (in the beam frame) Touschek scattering [4] was adjusted to account coupling. It assumes that the rms momentum spread is much smaller than the size of the RF bucket. The ratio $\sigma_{\mathrm{p}} /(\underline{\underline{\Delta}} / \mathrm{p})_{\text {sep }}$ was in the range of $[0.04,0.15]$ for the beam current changing in the $[0,4] \mathrm{mA}$. However, when $\sigma_{\mathrm{p}} /(\underline{\underline{\Delta}} / \mathrm{p})_{\text {sep }}$ achieves 0.15 this approximation becames not quite accurate. Therefore the corersponding 
correction was additionally computed.

In the coupled optics the only free parameter used in the modeling was the effective pressure of residual gas. To compute the effective pressere we used data for the uncoupled optics. A comparison of measurements with the model (see next section) determined the emittances set by the residual gas scattering: $\varepsilon_{1 \text { gas }} \approx \varepsilon_{2 \text { gas }} \approx$ $0.42 \mathrm{~nm}$. Note that the large contributions of SR into both transverse emittances resulted in that the gas scattering does not play any role on the beam parameters evolution with exception of the beam lifetime set by gas scattering at very small beam current when the Touschek scattering becomes negligible.

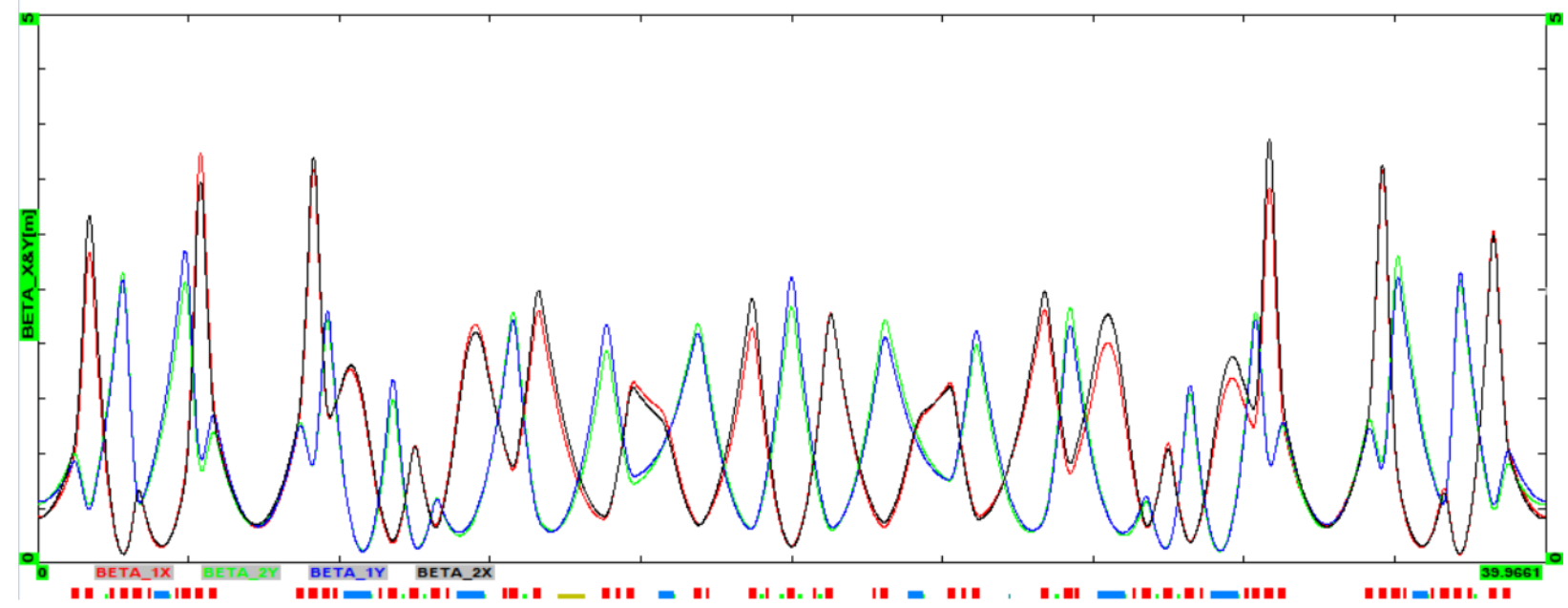

Figure 2: 4D beta-function for the coupled optics.

$\sigma \mathrm{x} \& \sigma_{\mathrm{y}}[\mathrm{mm}], \mathrm{L} 1$

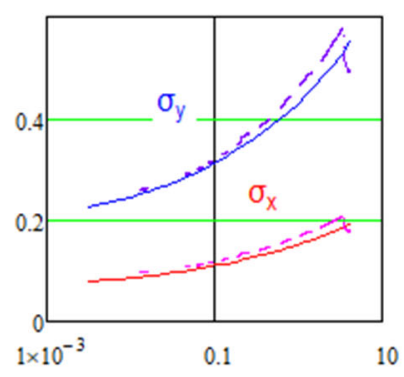

$\sigma \_\mathrm{x} \& \sigma_{\_} \mathrm{y}[\mathrm{mm}], \mathrm{L} 4$

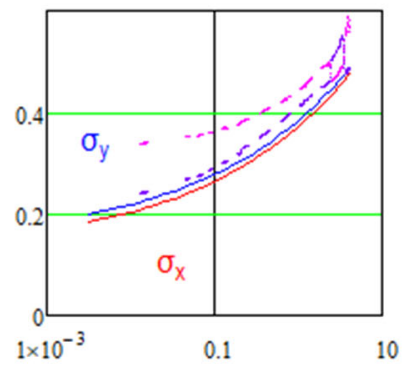

$\sigma \_\mathrm{x} \& \sigma \_\mathrm{y}[\mathrm{mm}], \mathrm{R} 3$

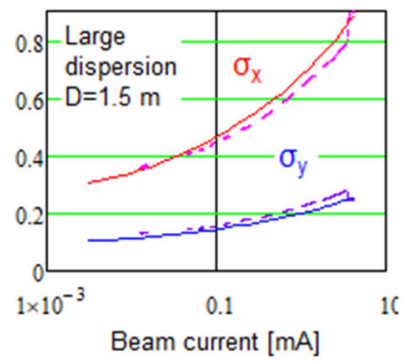

$\sigma \mathrm{x} \& \sigma \mathrm{y}[\mathrm{mm}], \mathrm{L} 2$

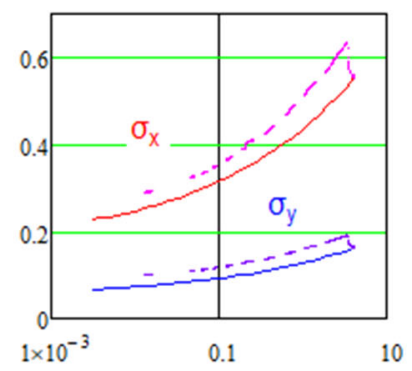

$\sigma \_\mathrm{x} \& \sigma \_\mathrm{y}[\mathrm{mm}], \mathrm{R} 1$

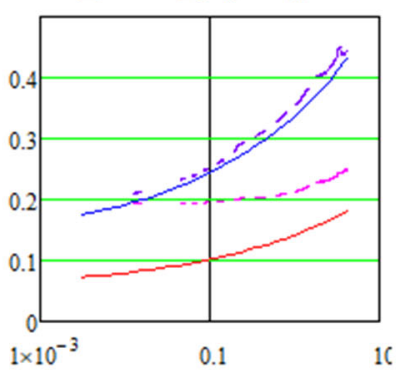

Momentum spread

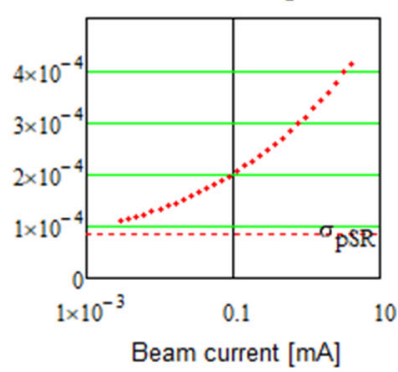

$\left.\sigma \mathrm{x} \& \sigma_{\mathrm{y}} \mathrm{\textrm {mm }}\right], \mathrm{L} 3$

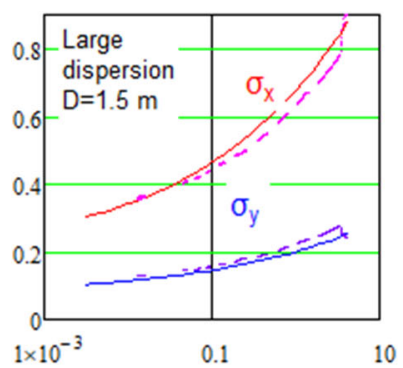

$\left.\sigma \_\mathrm{x} \& \sigma \_\mathrm{ym}\right], \mathrm{R} 2$

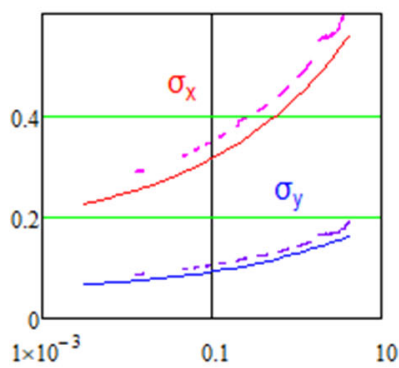

Emittances, nm

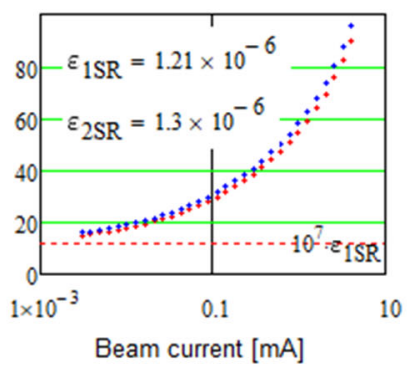

Figure 3: The measured (dashed lines) and predicted (solid lines) rms beam sizes at the synchrotron radiation monitors. 
Figure 3 shows the measured and predicted (solid lines) rms beam sizes at the synchrotron radiation monitors. As one can see the R1 camera looks poorly focused. The cameras L4, R1, R2 did not have gain control properly operating at the time of measurements and therefore their measurements may be compromised at large beam currents. Other cameras show decent coincidence between the measured and predicted beam sizes. There is a good coincidence for the high dispersion cameras (L3, R3). However, they still show smaller momentum spread at large beam current. The same as the WCM data the SR monitor shows smaller momentum spread.
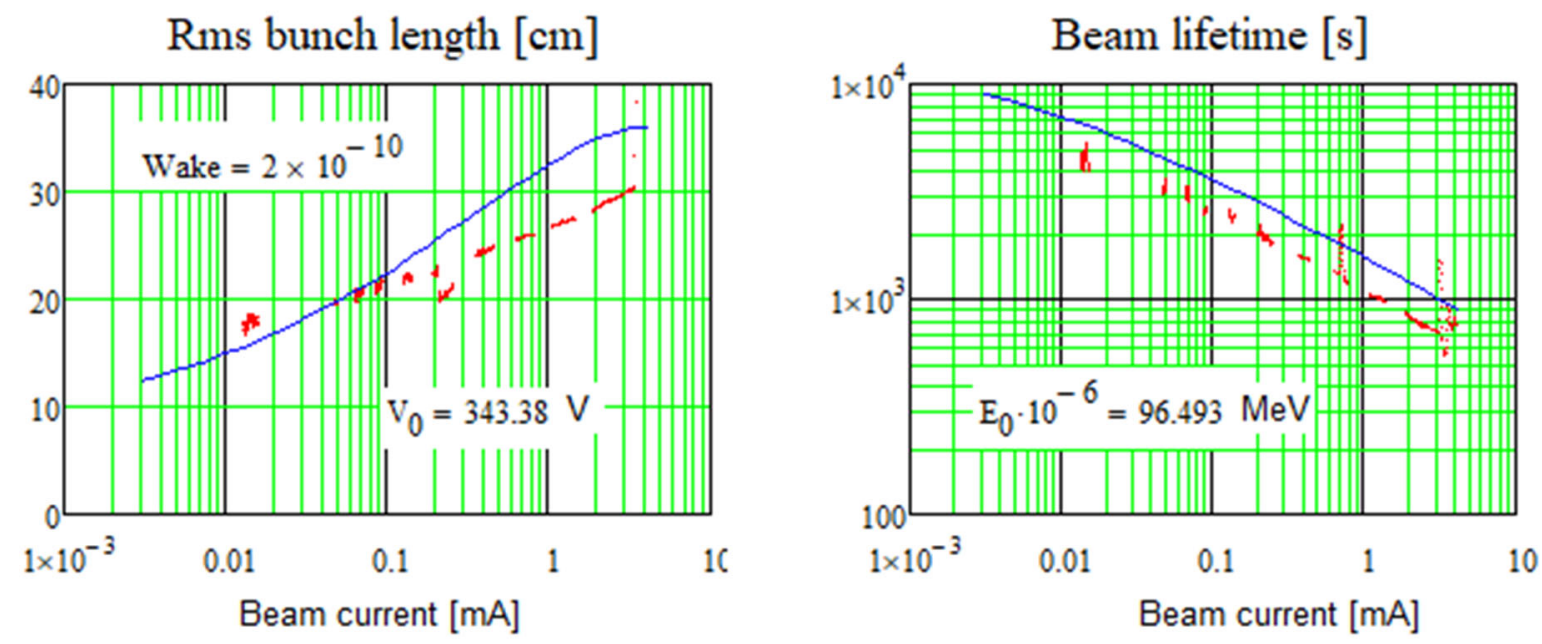

Figure 4: Predicted (blue) and measured (red) rms bunch length and beam lifetime as functions of the beam current.

The measured bunch length at small currents is larger than the model predicts. The most probable reason of this behavior is related to the phase noise of the RF voltage. It was not accounted in the simulations. To explain the observed discrepancy the RF phase noise of about $10^{-10} \mathrm{rad}^{2} / \mathrm{Hz}$ at the frequency of synchrotron motion is required. This is very small tough requirement. The corresponding measurements of RF noise are planned in the near future. The reason of the discrepancy at the large beam current is unknown. A possible reason is the beam longitudinal self-focusing due to ring impedance. Note also that there is some increase in the measured bunch length due to jitter in the scope triggering (multiple triggers are used for averaging) and the finite bandwidth of the scope which were not accounted in the computation of rms longitudinal beam size.

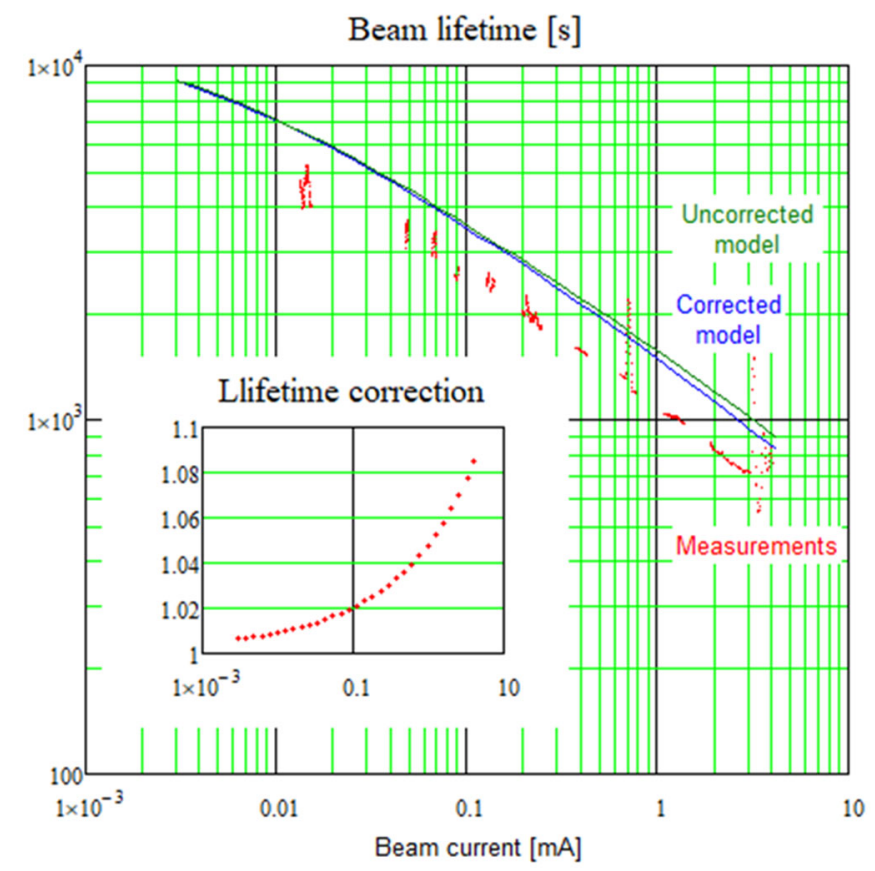

Figure 5:Correction to the Touschek scattering due to finite bunch length.

As one can see from Figure 4 the measured Touschek lifetime is $\sim 40 \%$ below model. Accounting the finite length of the bunch presented in Figure 5 reduces this difference to 35\%. The reason for the remaining 
discrepancy is unknown. The most probable reason is that a large momentum deviation of a particle results in a reduction of dynamic aperture and particle loss due to its transverse motion which is not accounted in the model.

Concluding we can state that for the coupled optics case:

- Calculated transverse beam sizes are quite close to the measured ones $(\sim 10 \%)$.

- There is considerable discrepancy for the bunch length

○ Measured lengths are longer at small current $(+10-20 \%)$

- Looks like there is a problem with the measurements and possibly a contribution from the RF phase noise

- Measured lengths are shorter at large current

- Looks like incorrect description of bunch self-compression which accounts bunch shortening due to beam interaction with vacuum chamber and its elements.

- Accuracy of transverse beam sizes at dispersive places is insufficient to extract the momentum spread with accuracy comparable to the bunch length measurements.

- The measured beam lifetime is $35 \%$ below predicted. The origin is unknown. There are no free parameters in the theory to change it. A study of particle loss due to aperture limitation at large momentum deviation can be the key in understanding of this phenomenon.

\section{MEASUREMENTS FOR THE CASE OF UNCOUPLED BEAM OPTICS}

There were three data sets acquired at March 6. The first two data sets were for the uncoupled motion and the last one for the coupled one. As on can see in Figure 6 there are dips in the bunch length just below $1 \mathrm{~mA}$. That is not observed in the coupled optics data. This dip also uncorrelates/correlates with the beam sizes. The origin of this dip is unknown. The most probable reason is an accumulation of ions in the beam but to verify or refute it additional theoretical and experimental studies are required. As one can also see the longitudinal stability is lost at the beam current above $3.3 \mathrm{~mA}$. We need to note that the above-mentioned dip is absent in March 17 data where the coupling and, consequently, the vertical beam size were larger.

\section{RMS bunch length $[\mathrm{cm}]$}

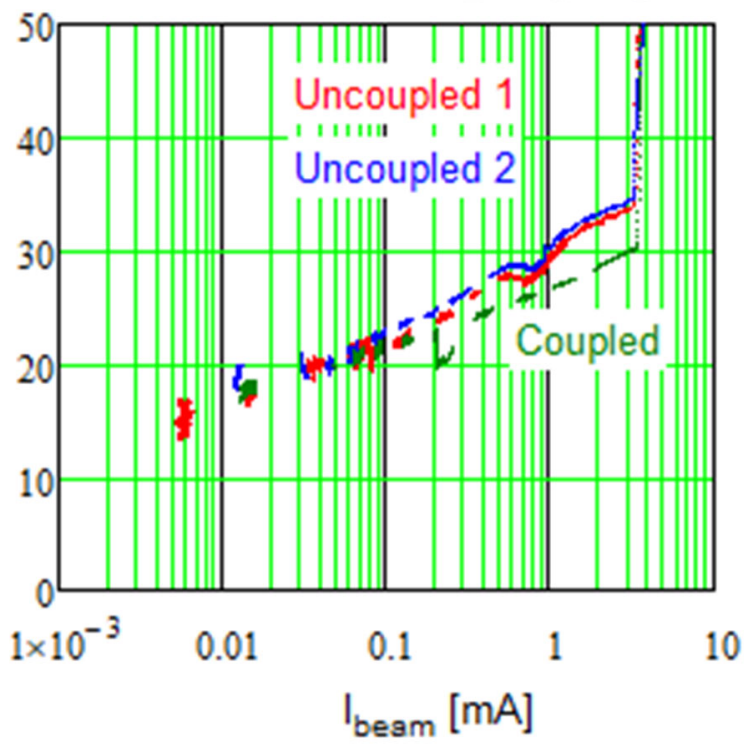

Figure 6:Measured rms bunch length on the beam current for the March 6 data.

The theoretical analysis used for the uncoupled optics measurements assumes uncoupled formalism in computation of single and multiple IBS. For multiple IBS the analysis assumes non-relativistic beam in the beam frame. It is not quite accurate at large beam current $(\sim 4 \mathrm{~mA})$. As one can see in Figure 7 at some locations 
the rms velocity achieves about half of the light speed. The full relativistic treatment [4] is used for computation of Touschek scattering.

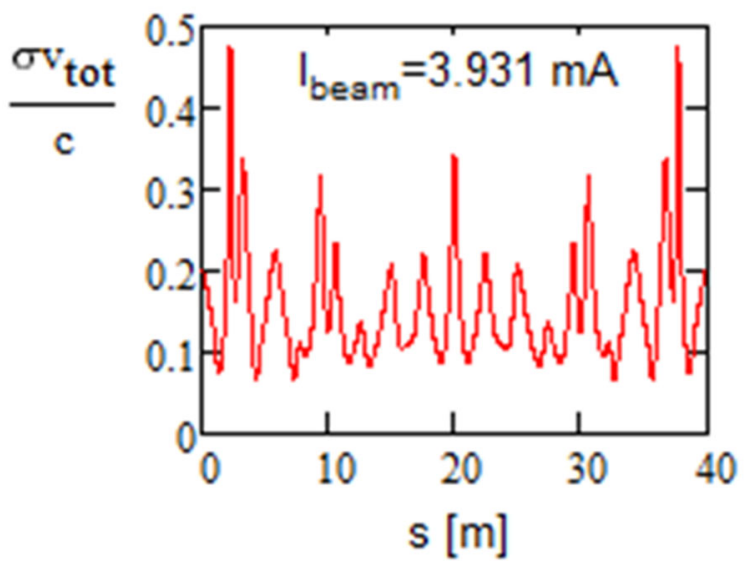

Figure 7: The rms velocity spread, $\beta=v / c$, in the beam frame along the IOTA ring for the beam current of $3.9 \mathrm{~mA}$.

$\sigma \_\mathrm{y}[\mu \mathrm{m}], \mathrm{L} 1$

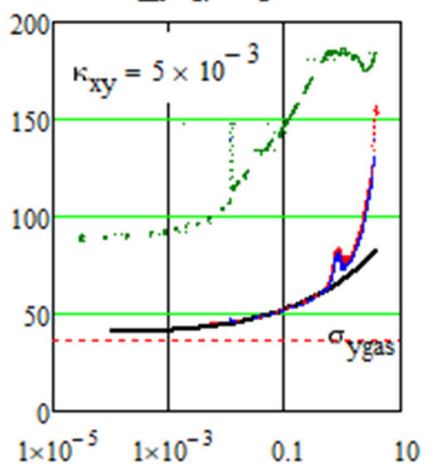

$\sigma \_\mathrm{y}[\mu \mathrm{m}], \mathrm{L} 4$

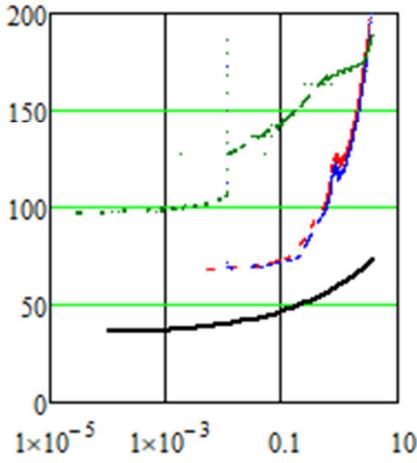

$\sigma \_\mathrm{y}[\mu \mathrm{m}], \mathrm{L} 2$

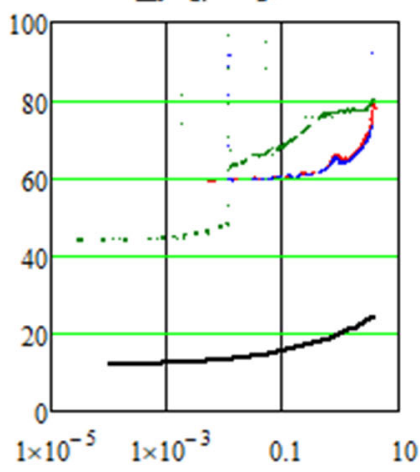

$\sigma \_\mathrm{y}[\mu \mathrm{m}], \mathrm{R} 1$

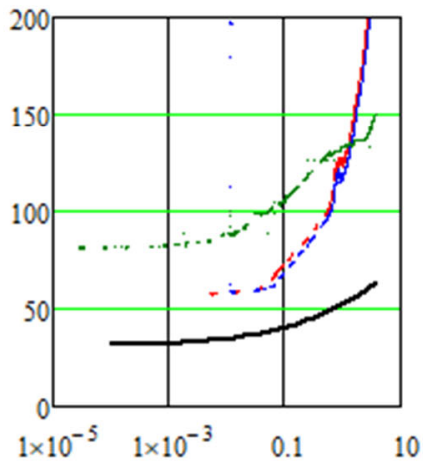

$\sigma \_\mathrm{y}[\mu \mathrm{m}], \mathrm{L} 3$

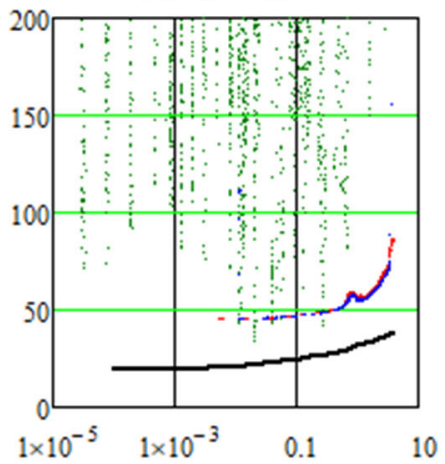

$\sigma \_\mathrm{y}[\mu \mathrm{m}], \mathrm{R} 2$

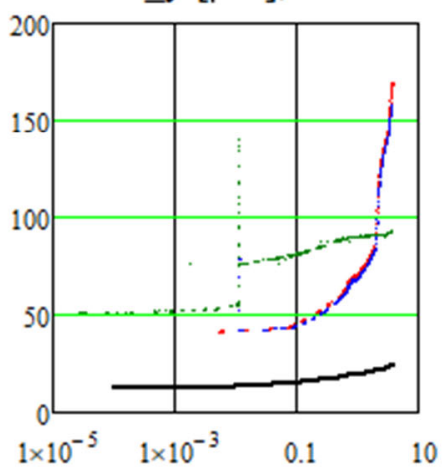

Model, $1^{\text {st }}$ March 6 meas., 2 ${ }^{\text {nd }}$ March 6 meas., March 17 meas., March 17 meas. Gaussian fitting

Figure 8: Dependence of vertical beam sizes on the beam current for uncoupled optics.

The same as for the coupled case in Touschek rate computation the momentum spread is assumed to be much smaller than the RF bucket height: $\sigma_{\mathrm{p}} /\left(\Delta \mathrm{p} / \mathrm{p}_{\text {sep }} \in[0.04,0.15]\right.$ for the beam current in the range $[0,4]$ $\mathrm{mA}$. The effective pressure of residual gas plays major role at the beam small current. Together with SR it sets the vertical emittance. The gas contribution to the emittance is: $\varepsilon_{\mathrm{xgas}} \approx \varepsilon_{\mathrm{ygas}} \approx 0.42 \mathrm{~nm}$. Residual gas scattering sets the beam lifetime to $\sim 200 \mathrm{~min}$. In the absence of coupling for both SR and IBS $d \varepsilon_{y} / d t=0$. Strictly speaking the coupling coefficients for the IBS and SR are different. However, taking into account limited knowledge about coupling details we assume that both coupling coefficients are equal: $d \varepsilon_{y} / d t=\kappa d \varepsilon_{x} / d t$, where $\kappa<<1$ is the coupling coefficient. The coupling coefficient can be uniquely determined at large beam 
current where contribution of the residual gas scattering is negligible.

Figure 9 shows the vertical beam sizes for the uncoupled optics. The smallest $\varepsilon_{\mathrm{y}}$ is achieved at measurements of the L1 SR monitor for the both March 6 measurements. The March 17 measurements had larger coupling and therefore were ignored in the analysis. Comparison of the model and the measurement results at large currents yields the coupling coefficient: $\kappa=5 \cdot 10^{-3}$. Comparison at small currents yields $\varepsilon_{y_{\text {gas }}}=0.42 \mathrm{~nm}$. The actual value should be somewhat smaller due to diffraction contribution to the SR monitor measurements which theoretical value is about $20 \mu \mathrm{m}$. We do not have an experimental verification of this value. There is fast emittance growth above $\sim 0.5 \mathrm{~mA}$. It looks as an effect of stored ions.

$\sigma_{-} \mathrm{x}[\mathrm{mm}], \mathrm{L} 1$

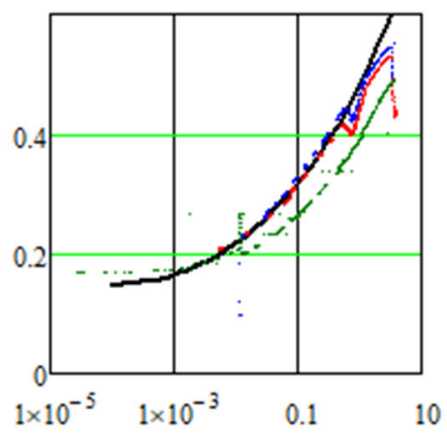

$\sigma_{-} \mathrm{x}[\mathrm{mm}], \mathrm{L} 4$

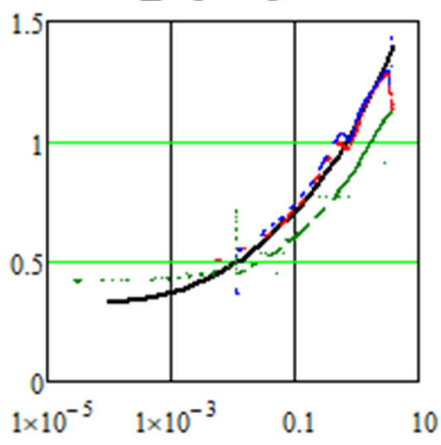

$\sigma_{-} \mathrm{x}[\mathrm{mm}], \mathrm{L} 2$

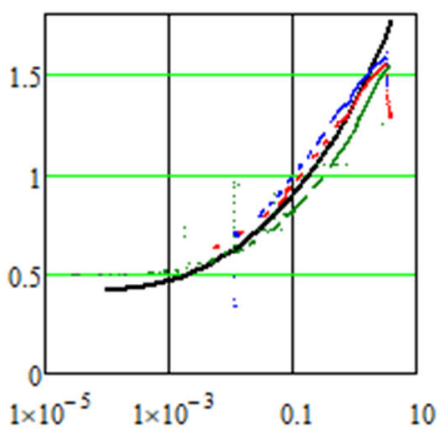

$\sigma_{-} \mathrm{x}[\mathrm{mm}], \mathrm{R} 1$

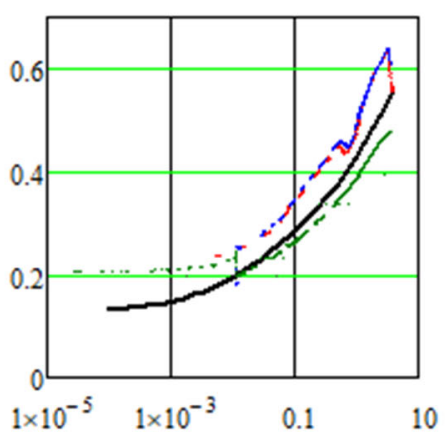

$\sigma_{-} \mathrm{x}[\mathrm{mm}], \mathrm{L} 3$

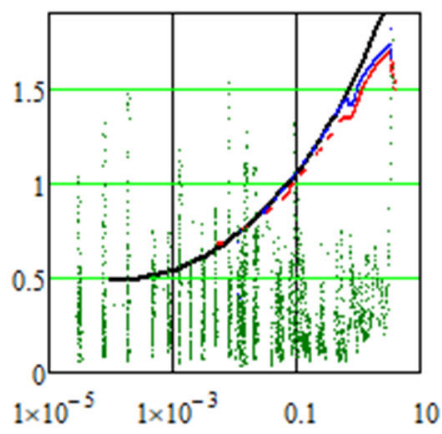

$\sigma_{-} \mathrm{x}[\mathrm{mm}], \mathrm{R} 2$

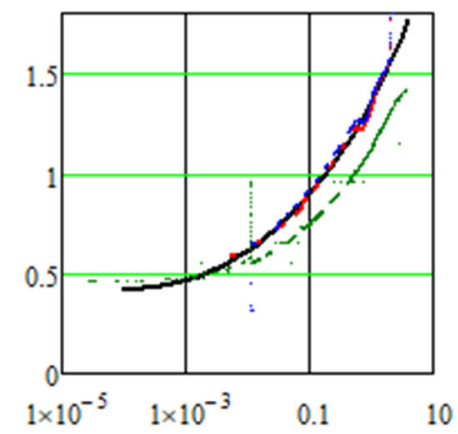

Model, $1^{\text {st }}$ March 6 meas., $2^{\text {nd }}$ March 6 meas., March 17 meas., March 17 meas. Gaussian fitting

Figure 9: Dependence of horizontal beam sizes on the beam current for uncoupled optics.

Figure 9 shows the horizontal beam sizes for uncoupled optics. There is good agreement for the March 6 data, while the March 17 data show smaller $\varepsilon_{\mathrm{x}}$ because larger value of $\varepsilon_{\mathrm{y}}$ due to larger coupling. The dip in the horizontal emittance above $0.5 \mathrm{~mA}$ anticorrelates with the bump in the vertical emittance and correlates with the dip in the bunch length. Looks like that the vertical emittance increase due to ions results in a reduction of IBS with subsequent reduction of horizontal and longitudinal emittances.

Figure 10 shows the beam lifetime and the bunch length for the uncoupled optics. The same as for the coupled measurements the measured lifetime is $\sim 30 \%$ smaller at large beam current for March 6 data where the model parameters were tuned. The predicted bunch length is shorter at small currents and larger at the large currents. At the large beam currents the bunch length computed with the Gaussian fit is even shorter. That points out that there are non-Gaussian tails.

\section{EFFECTIVE VACUUM}

On the first glance there are two independent ways to obtain the effective vacuum: first - from the beam lifetime at the small beam current, second - from the minimum measured vertical emittance at the small beam current. However, corresponding computations exhibited the results which are different by almost two orders of magnitude. The detailed analysis showed that only the first way of computations yields the correct value. The problem with the second way, which is based on the equilibrium emittance, is related to the distribution shape which becomes non-Gaussian and has long non-Gaussian tails which are ignored on the computation of 
the measured rms beam size. The reason of such behavior is that only few gas scattering events happen during one SR damping time. That generates distribution with small bright spot in the center and very long tails.

The following equation,

$$
\tau_{\text {gas }}^{-1}=\frac{2 \pi c r_{e}^{2}}{\gamma^{2} \beta^{3}}\left\langle\left(\frac{\beta_{x}(s)}{\varepsilon_{x m}}+\frac{\beta_{y}(s)}{\varepsilon_{y m}}\right) n_{e f f}(s)\right\rangle_{s}, \quad n_{e f f}(s)=\sum_{\substack{k \\ \text { overatoms }}} Z_{k}\left(Z_{k}+1\right) n_{k}(s),
$$

was used in the computations of the effective gas pressure. Here $r_{\mathrm{e}}$ is the electron classical radius, $<>_{\mathrm{s}}$ denotes averaging over the ring, $\varepsilon_{\mathrm{mx}}$ and $\varepsilon_{\mathrm{my}}$ are the ring acceptances, $\beta_{\mathrm{x}}$ and $\beta_{\mathrm{y}}$ are the beta-functions, and summing is performed over gas species. For estimate we assume a uniform gas distribution along the ring. Other parameters are: the measured acceptances $-\varepsilon_{\mathrm{xm}}=22 \mu \mathrm{m}, \varepsilon_{\mathrm{ym}}=40 \mu \mathrm{m}$, and averaged beta-functions $-\beta_{\mathrm{xa}}=2.16 \mathrm{~m}$, $\beta_{\mathrm{ya}}=1.94 \mathrm{~m}$. That yields the effective pressure $\mathrm{P}_{\mathrm{eff}}=4.2 \cdot 10^{-8}$ Torr of atomic hydrogen equivalent for the measured beam life of $200 \mathrm{~min}$. This value is close to what we should expect for non-baked vacuum chamber. Using this $\mathrm{P}_{\mathrm{eff}}$ and the following expression,

$$
\frac{d \varepsilon_{y}}{d t}=\frac{\varepsilon_{y}}{2 \tau_{y S R}}=\frac{2 \pi c r_{e}^{2}}{\gamma^{2} \beta^{3}}\left\langle\beta_{y}(s) n_{e f f}(s)\right\rangle_{s}, \quad n_{\text {eff }}(s)=\sum_{\begin{array}{c}
k \\
\text { over atoms }
\end{array}} Z_{k}\left(Z_{k}+1\right) n_{k}(s) \ln \left(\frac{204}{\sqrt[3]{Z_{k}}}\right),
$$

we obtain the equilibrium vertical emittance of $5.6 \mathrm{~nm}$. That is 13 times larger than $0.42 \mathrm{~nm}$ measured. Actually, the difference should be even larger because we should subtract the diffraction contribution from the vertical beam size image of the L1 synchrotron radiation monitor.
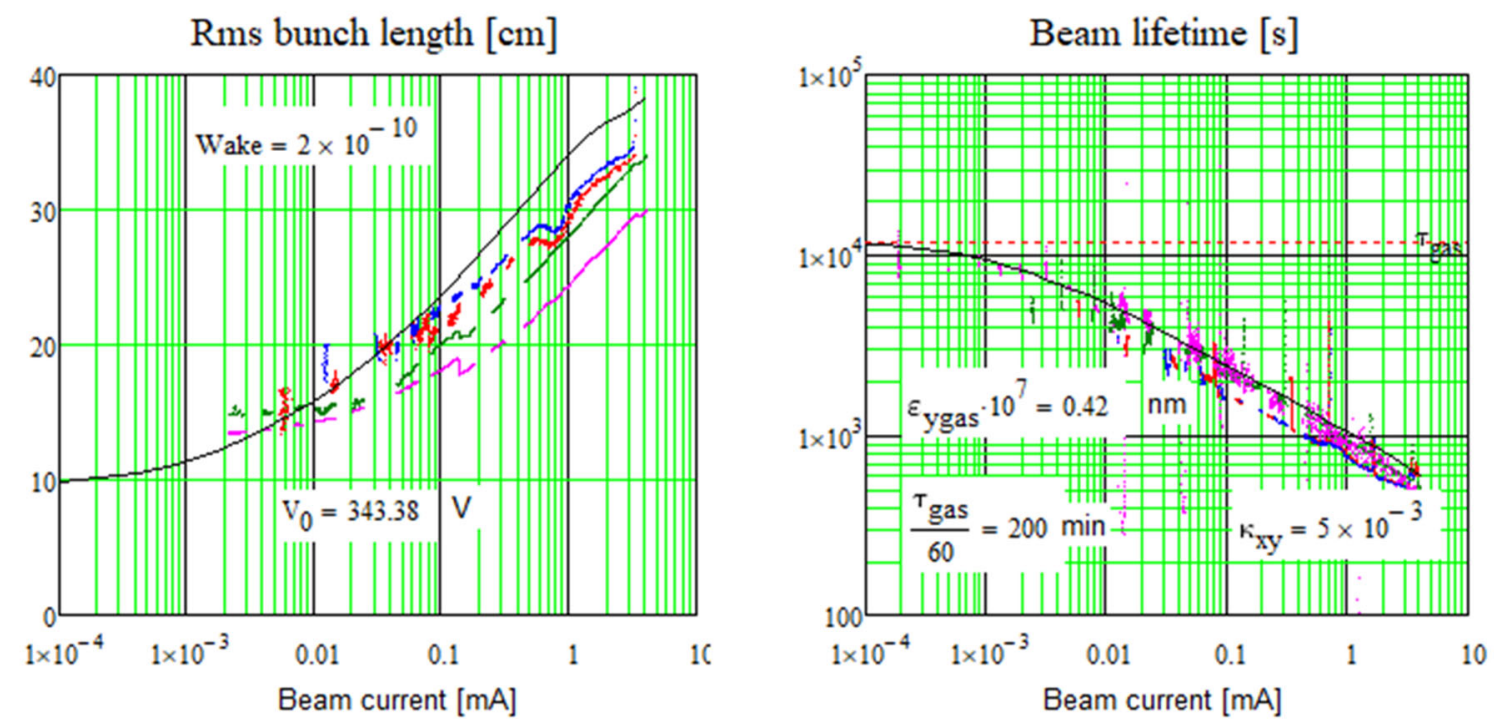

Model, $1^{\text {st }}$ March 6 meas., 2 ${ }^{\text {nd }}$ March 6 meas., March 17 meas., March 17 meas. Gaussian fitting

Figure 10: Dependence of the beam lifetime and the bunch length on the beam current for the uncoupled optics.

\section{CONCLUSIONS}

Data analysis showed reasonable comparison between the measured and predicted parameters. However, many questions are still not answered and additional measurements should be planned in the future.

For both the coupled and uncoupled optics the measurements show $\sim 30-40 \%$ smaller lifetime than the model predicts. The reasons are still unknown.

The measurements carried out after the study showed that a longer bunch length measured for short bunches is related to the bandwidth limitation of the scope, the jitter in its triggering and very probably to the RF phase noise.

For the coupled optics the model predicts well both emittances. For uncoupled optics the horizontal emittance is predicted well for the beam currents below $0.5 \mathrm{~mA}$. However, at larger currents there are considerable discrepancies. It looks like that we have ions stored in the beam. To make sure that it is the case we need an independent verification. 
These IBS measurements were extremely helpful in the preparation of IOTA for the coming OSC run. They showed that the instrumentation is mostly ready. However, the present bunch length measurements based on the wall current monitor cannot be used for the bunch length measurements at the OSC where the bunch length is expected to be more than two times shorter. The achieved resolution of the sync-light monitors of $\sim 50 \mu \mathrm{m}$ is insufficient for accurate measurements of the OSC beam size which in the case of strong OSC goes down to 5-10 $\mu \mathrm{m}$. Measurements also proved that a significant vacuum improvement is required for the OSC run.

\section{ACKNOWLEDGMENTS}

The authors would like to thank the entire Accelerator Science and Technology Department of Fermilab Accelerator division for support of the IOTA day-to-day operation, repairs and developments. In particular, we are grateful to K. Carson and D. Edstrom for technical support.

\section{REFERENCES}

[1] Valeri Lebedev, "Understanding Lifetimes, Beam Sizes and Gas Scattering in IOTA", https://indico.fnal.gov/event/43231/contributions/187359/attachments/129996/158058/IBSatIOTAcolab orMeeting.pdf

[2] V. Lebedev, S. Bogacz, e-print JLAB-ACC-99-19 (1999), JINST, v. 5, (2010)

[3] V. Lebedev, S. Nagaitsev, "Multiple intrabeam scattering in X-Y coupled focusing systems", https://arxiv.org/abs/1812.09275

[4] "Handbook of Accelerator Physics and Engineering", 2-nd edition, edited by A. Chao, K. Mess, M. Tigner and F. Zimmermann (2013) 